\title{
Accuracy of CBCT Linear Measurements in Orthogonal versus Corrected Coronal Planes
}

\author{
Remy Golding ${ }^{1 *}$, Laurence J Walsh ${ }^{2}$, Paul Monsour ${ }^{3}$ \\ ${ }^{1}$ Student, DClinDent (DMFR) Program, University of Queensland Dental School, 288 Herston Rd, Herston, \\ Queensland, Australia \\ ${ }^{2}$ Emeritus Professor, University of Queensland, Australia
}

${ }^{3}$ Emeritus Professor, Foundation Chair for Dento-Maxillofacial Radiology, University of Queensland, Australia

*Corresponding Author: Remy Golding, Student, DClinDent (DMFR) Program, University of Queensland Dental School, 288 Herston Rd, Herston, Queensland, Australia; Email: remy.golding @ gmail.com

Received Date: 07-07-2021; Accepted Date: 22-07-2021; Published Date: 29-07-2021

Copyright $^{\odot} 2021$ by Golding R, et al. All rights reserved. This is an open access article distributed under the terms of the Creative Commons Attribution License, which permits unrestricted use, distribution and reproduction in any medium, provided the original author and source are credited.

\begin{abstract}
Objectives: To assess the accuracy of measurements between a simulated tooth root and Inferior Dental Canal (IDC) in medium field-of-view Cone Beam Computed Tomography (CBCT) scans, comparing orthogonal plane measurements with views 'corrected' perpendicular to the IDC.
\end{abstract}

Study Design: Sixteen CBCT scans were acquired, including equally $0.2 \mathrm{~mm}$ and $0.4 \mathrm{~mm}$ resolutions and two machines. Distances between root and IDC of 1-3 mm (horizontal and vertical) and $0.5 \mathrm{~mm}$ (horizontal) were measured with Radiant DICOM viewer. Retest reliability was assessed for 80 sites.

Results: High retest reliability was demonstrated (limits of agreement $-0.33 \mathrm{~mm}[(-0.42)-(-0.25)]$ to $0.28 \mathrm{~mm}[0.19-0.36])$. Uncorrected maximum over-estimation was $0.74 \mathrm{~mm}$, and median was -0.02 $\mathrm{mm}$ (SD 0.21). Corrected maximum over-estimation was $0.64 \mathrm{~mm}$, and median was $-0.04 \mathrm{~mm}$ (SD 0.19 ). The difference in medians was found to be statistically significant ( $p=0.006, d=0.26$ ). Uncorrected measurements were larger than corrected by greater than $0.5 \mathrm{~mm}$ in seven instances (maximum difference of $0.77 \mathrm{~mm}$ ), and smaller by greater than $0.5 \mathrm{~mm}$ in two instances (maximum difference of $0.64 \mathrm{~mm}$ ).

Conclusions: Idealised CBCT measurements can achieve sub-millimetre accuracy. Corrected view measurements may reduce the risk of distance over-estimation. Further prospective research using patient data is warranted.

Golding R | Volume 2; Issue 2 (2021) | JDHOR-2(2)-029 | Research Article 


\section{Keywords}

Cone-Beam Computed Tomography; Dimensional Measurement Accuracy; Mandibular Nerve; Dentistry; Radiology

\section{Introduction}

Panoramic Radiography (PR) is often the primary radiographic technique for pre-operative surgical assessment in the posterior mandible [1,2]. While PR alone may provide sufficient radiographic information to proceed with treatment in some cases, PR may not always adequately demonstrate the relevant anatomy [3,4]. In such cases, further three-dimensional imaging may be justified, including Cone Beam Computed Tomography (CBCT) $[5,6]$.

Dental and maxillofacial applications of CBCT were first described by Mozzo, et al., in 1998 [7]. Since then, CBCT has become an increasingly popular alternative to fan-beam CT for certain applications, with a vast array of CBCT machines subsequently coming to market [8].

While the size of the reconstructed three-dimensional elements (voxels) in a CBCT dataset may be as small as $0.076 \mathrm{~mm}$, a number of patient and machine factors influence the acquired data $[8,9]$. Brüllmann and Schulze note that, after accounting for scatter and patient movement, "a realistic spatial resolution of just above one line pair per millimetre could be expected" [10]. Should the subsequent display and viewing conditions be suboptimal, including an adequate monitor and lighting conditions, this can further detract from the diagnostic quality of the image [11].

Where measurements of linear distances are to be made using CBCT datasets, a number of other factors must also be considered. The user's ability to perceive and select appropriate landmarks is an important factor, and is affected by factors such as partial volume averaging between voxels [12]. Additionally, the software may then measure "the distance between the midpoints of the voxels" in such a way that "half of the voxel would not be included in the measurement on either side" [12].

The risks associated with distance over-estimation may be more severe than those associated with under-estimation. Where the space between a landmark and a vital structure is overestimated, surgical over-extension may cause damage to vital structures. On this basis, safety margins up to $2 \mathrm{~mm}$ have been advocated in the surgical application of linear measurements from CBCT data [13]. 
Notably, as anatomic structures generally course obliquely to the orthogonal planes, an orthogonal slice is unlikely to demonstrate the points where two structures are at their closest. Subsequently, measurements made in the orthogonal planes may over-estimate the available space. Several previous imaging studies describe measurements taken from slices which have been aligned or 'corrected' to match the path of the Inferior Dental Canal (IDC) $[1,14,15]$. However, no direct comparison of measurement accuracy has been made of orthogonal versus corrected views.

This study was designed to investigate the accuracy of measurements between a simulated tooth root and IDC in manufactured models of known dimensions, comparing measurements taken in a standard coronal view with measurements from a view aligned or 'corrected' perpendicular to the simulated IDC. Measurements taken in both orientations were further compared with the dimensional specifications used for milling of the model, as the 'gold standard'.

\section{Materials and Methods}

Plastic models were created through computer-assisted design and manufacture to represent multiple tooth roots at pre-determined distances from the simulated IDC. Digital models were generated in the Solidworks eDrawings system (Dassault Systèmes SolidWorks Corporation, 175 Wyman St, Waltham, Massachusetts, USA). Clear acrylic of radiodensity greater than water was selected for the base components of the model, and a grey Polyvinyl Chloride (PVC) of higher radiodensity was selected to represent both the IDC cortical borders and tooth roots.

The models were milled using a calibrated HAAS Lathe with SL30 attachment (CNC Machines, 103/111 Central Park Place, Sanford, Florida, USA) with a reported displacement tolerance of less than $0.005 \mathrm{~mm}$. Following assembly, the 'friction fit' between the components prevented any disassembly. A fit-check CBCT scan obtained at $0.076 \mathrm{~mm}$ resolution and maximal exposure settings demonstrated no visible air gap, distortion or fracture.

The model design is demonstrated in Fig. 1.

Five coronal cross-sectional configurations were included in the block design, each with two root-to-IDC relationships.

At cross-sections 1, 2 and 3, one root was positioned superior and one root lateral to the IDC, at distances of 1,2 and $3 \mathrm{~mm}$, respectively. 
At cross-section 4 and 5, the IDC was centrally positioned between root apices to either side, with different supero-inferior positioning. At cross-section 4, the inferior extent of the apices was level with the centre of the IDC, at a distance of $1.0 \mathrm{~mm}$.

At cross-section 5, the IDC passed horizontally between the roots above the level of the apices, at a distance of $0.5 \mathrm{~mm}$. In total, each model contained seven horizontal and three vertical rootto-IDC relationships for measurement.

Four wedge-shaped positioning jigs were fabricated from polyvinylsiloxane (PVS) by holding the model in a position simulating the angulation of the IDC with a patient in anatomic position, while the material was allowed to set. The positioning jigs were stabilised into the base of a water-filled plastic container of $120 \mathrm{~mm}$ diameter, designed to simulate scatter from patient tissues, and the apparatus positioned within the CBCT machine. The resultant angulation of the model in the coronal, axial and sagittal views is demonstrated in Fig. 2.

Eight CBCT datasets were acquired with each of two CBCT units, namely the iCAT FLX V17 (Imaging Sciences International, 1910 N Penn Rd, Hatfield, Philadelphia, USA) and ProMax 3D Max (Planmeca, Asentajankatu 6, Helsinki, Finland). Datasets were acquired at both 'normal resolution' $(0.2 \mathrm{~mm})$ and 'low resolution' $(0.4 \mathrm{~mm}$ resolution $)$ in each machine, with volumes of $13 \times 5.5 \mathrm{~cm}$ at $96 \mathrm{kVp}$ for the Planmeca, and $16 \times 6 \mathrm{~cm}$ at $120 \mathrm{kVp}$ for the iCAT. The apparatus remained in the same position for the acquisition of low and normal resolution scans for each positioning jig.

The resulting DICOM datasets were measured by the lead researcher in RadiAnt DICOM viewer (Medixant, Promienista 25, 60-288 Poznań, Poland) with a 32 UJ590 UHD monitor (Samsung Electronics, 11, Seocho-daero 74-gil, Seocho, Seoul, South Korea). Room lighting was provided by two VBLFP-BL-312-SM surface-mounted dimmable lights (Vibe Lighting, Sydney, Australia; http://www.vibelighting.com.au), adjusted to minimise monitor spectral reflection.

As integer-based selection of magnification was not available within the software, an approximate magnification of 10x was set manually, by matching a $25 \mathrm{~mm}$ line in the dataset with a $250 \mathrm{~mm}$ ruler on the monitor. Measurements within each dataset were taken sequentially proceeding from cross-section 1 through 5 in the uncorrected/orthogonal coronal view, before aligning the IDC cortical border with the sagittal and axial planes to create the corrected coronal view (Fig. 2). During placement of line points, were the length value box to be within the observer's line of sight, the value box was moved out of sight, and the points both readjusted. Three individual measurements were taken with this method, and the median of the three measures recorded. 
Uncorrected and corrected view measurements were denoted ' $\mathrm{UN}$ ' and 'COR', respectively. Over-estimation of both uncorrected and corrected view measurements were calculated, and denoted 'OU' and 'OC', respectively. Instances of distance under-estimation were represented by negative $\mathrm{OU}$ or $\mathrm{OC}$ values. The difference in over-estimation between paired uncorrected and corrected measurements was calculated as the former minus the latter, and denoted 'DIF'. The absolute error of uncorrected and corrected measurements were also calculated, and denoted 'EU' and 'EC'. All instances of over- or under-estimation greater than or equal to 0.5 $\mathrm{mm}$ were identified for further assessment.

Data recorded for each set of measurements also included the machine type, scan number (cluster), scan resolution, quadrant (left or right), measurement direction (horizontal or vertical) and side of the IDC relative to the root (IDC-buccal, IDC-lingual or IDC-inferior). After one week, intra-observer retest measurements were obtained for 40 sites across four datasets.

Statistical analyses were undertaken primarily in the Jamovi software package. Normality of distribution was assessed using the Shapiro-Wilk method. The effect of independent variables with two groups was assessed using Wilcoxon-Rank paired or Mann-Whitney U T-tests. The effect of independent variables with three or more groups was assessed by one-way ANOVA on ranks (Kruskall-Wallis) with post-hoc pairwise comparisons using the Dwass-SteelCritchlow-Fligner method. For comparisons involving distances and direction, only data from cross-sections 1-3 were included, to eliminate the possibility of confounding interactions. Testretest reliability was assessed by Bland-Altman analysis. Intra-observer reliability was not assessed.
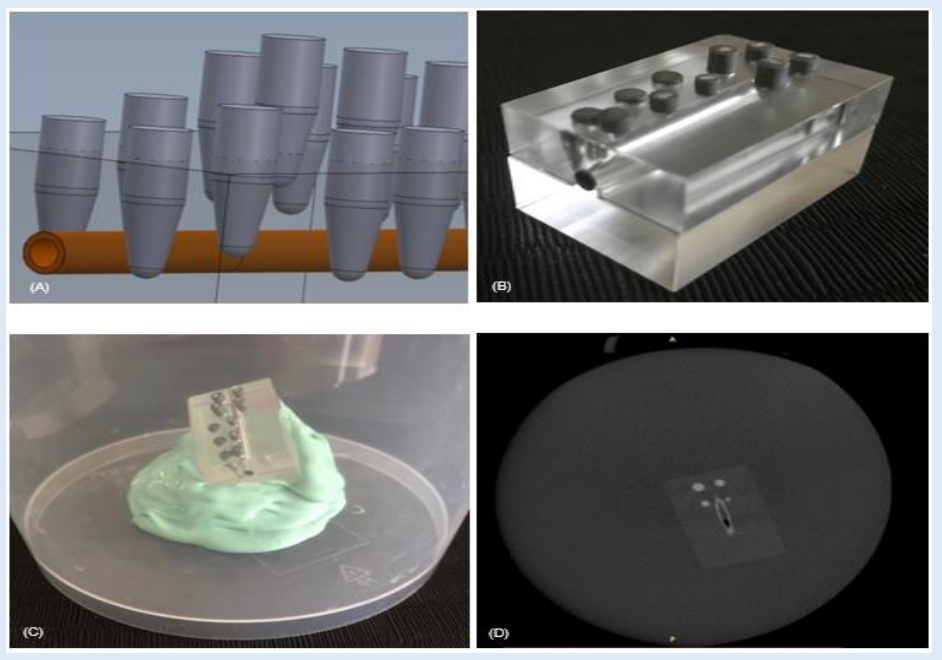

Figure 1: Model design and assembled apparatus. (A) Computer model prototype, demonstrating roots and canal; (B) Assembled model; (C) Model within positioning jig and container (without water); (D) High axial view of scanned model, intersecting more superiorly-positioned posterior roots. 

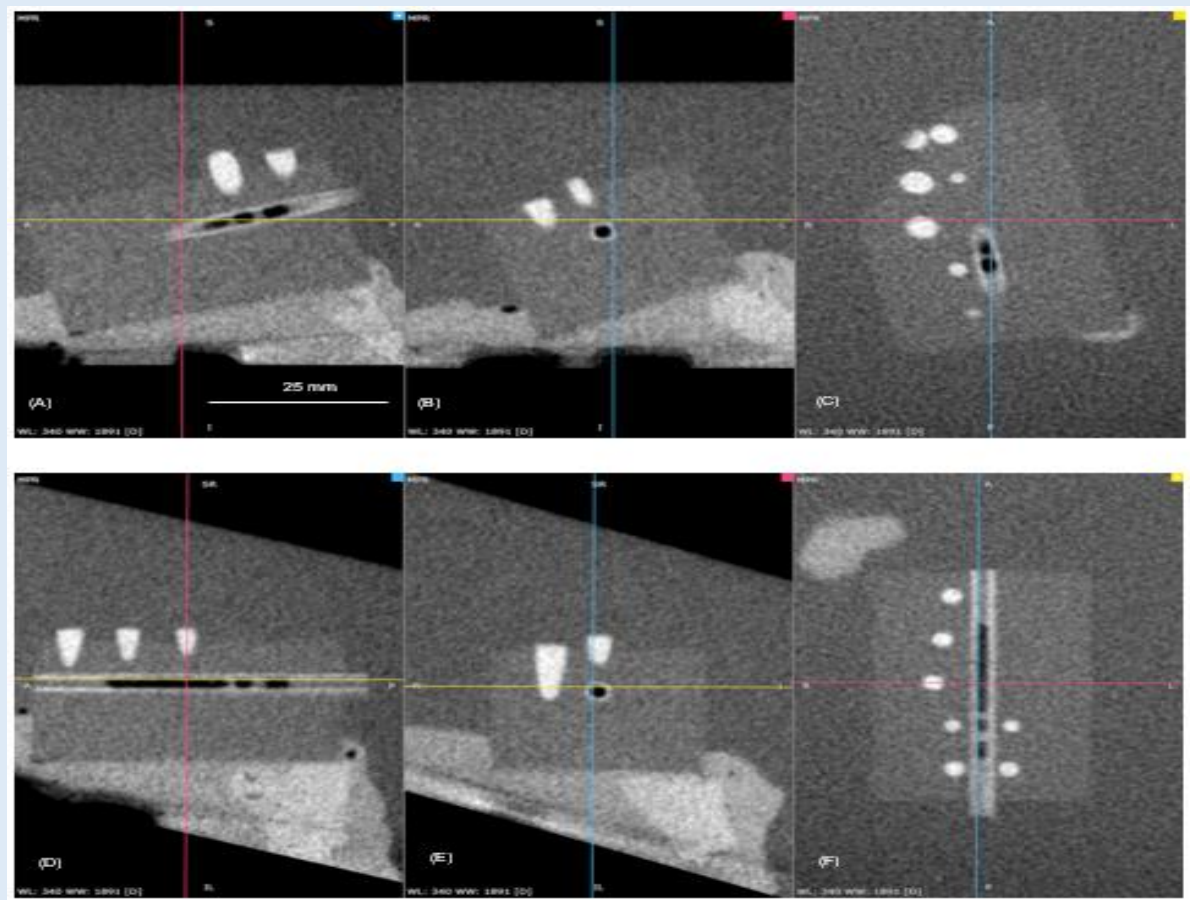

Figure 2: Example measurement views, at 10x screen magnification. (A) Scale for figure provided; (A-C) Uncorrected sagittal, coronal and axial views; (D-F) Corrected sagittal, coronal and axial views.

\section{Results}

Median EU was $0.10 \mathrm{~mm}$ (SD 0.15) and median EC was $0.12 \mathrm{~mm}$ (SD 0.13). Median OU was $-0.04 \mathrm{~mm}$ (SD 0.19) and median OC was $-0.04 \mathrm{~mm}$ (SD 0.19). Median values and differences for $\mathrm{UN}$ and $\mathrm{COR}$ at each distance are summarised in Table 1. The violin plot in Fig. 3 demonstrates the distribution of OU and OC. High intra-observer reliability was demonstrated by Bland-Altman analysis, with limits of agreement of $-0.33 \mathrm{~mm}(-0.42--0.25)$ to $0.28 \mathrm{~mm}$ (0.19- 0.36).

Overall, measurements were smaller for iCAT versus Planmeca. Differences were statistically significant for both UN $(0.12,95 \%$ CI $0.18-0.06, p<0.001, d=0.36)$ and COR $(0.13,95 \%$ CI $0.08-0.18, \mathrm{p}<0.001, \mathrm{~d}=0.41)$. Statistically significant differences in UN and COR occurred only between iCAT and Planmeca scans (never between same-machine scans), with median iCAT measures being smaller than for Planmeca for all scans.

Both UN and COR were larger at $0.2 \mathrm{~mm}$ resolution versus $0.4 \mathrm{~mm}$, with median differences of $0.08 \mathrm{~mm}(95 \%$ CI $0.02-0.14, \mathrm{p}<0.01)$ and $0.12 \mathrm{~mm}(95 \%$ CI $0.03-0.13, \mathrm{p}<0.01)$. Within the even proportion of horizontal and vertical measurements at 1-3 $\mathrm{mm}$ in cross-sections $1-3$, horizontal measurements tended to be larger than vertical, with median differences of $0.11 \mathrm{~mm}$

Golding R | Volume 2; Issue 2 (2021) | JDHOR-2(2)-029 | Research Article

Citation: Golding R, et al. Accuracy of CBCT Linear Measurements in Orthogonal versus Corrected

Coronal Planes. J Dental Health Oral Res. 2021;2(2):1-14.

DOI: http://dx.doi.org/10.46889/JDHOR.2021.2204 
for UN (95\% CI $0.03-0.21, \mathrm{p}<0.001, \mathrm{~d}=0.32)$ and $0.11 \mathrm{~mm}$ for COR (95\% CI $0.04-0.19$, $\mathrm{p}=<0.01, \mathrm{~d}=0.36$ ). No statistically significant effect was found for quadrant on UN or COR $(\mathrm{p}=0.71$ and $\mathrm{p}=0.86$, respectively).

The overall difference between UN and COR was found to be statistically significant ( $\mathrm{p}=$ $0.006, d=0.26)$. While differences at $3.0 \mathrm{~mm}$ were statistically significant $(\mathrm{p}<0.001, \mathrm{~d}=$ $0.72)$, differences at $0.5 \mathrm{~mm}, 1.0 \mathrm{~mm}$ and $2.0 \mathrm{~mm}$ did not reach statistical significance $(\mathrm{p}=$ $0.67, \mathrm{p}=0.18$, and $\mathrm{p}=0.84$, respectively).

The overall difference in absolute error between UN and COR was not significantly different $(\mathrm{p}=0.81)$, with statistically significant differences noted only at $2.0 \mathrm{~mm}(\mathrm{p}=0.02, \mathrm{~d}=0.52)$ and at $3.0 \mathrm{~mm}(\mathrm{p}=0.01, \mathrm{~d}=0.55)$.

Of the seven individual measurements which over-estimated distances by $0.5 \mathrm{~mm}$ or more, all were horizontal Planmeca measurements, with 5/7 (71.4\%) uncorrected and 5/7 (71.4\%) at 0.4 $\mathrm{mm}$ resolution. These over-estimations occurred across all distance groups, accounting for $5 / 160(3.1 \%)$ of uncorrected and 2/160 (1.3\%) of corrected measurements. All three distance under-estimations exceeding $0.5 \mathrm{~mm}$ were corrected vertical measurements at distances of 3 $\mathrm{mm}$ within two iCAT datasets, accounting for 3/160 (1.9\%) of corrected measurements. Of these, $2 / 3(66.7 \%)$ were at $0.4 \mathrm{~mm}$ resolution.

Among paired measurements, $\mathrm{UN}$ was greater than COR by $0.5 \mathrm{~mm}$ or more in seven instances, with a maximum difference of $0.77 \mathrm{~mm}$. Among these pairs, median OU was $0.62 \mathrm{~mm}$ (SD 0.23 ) and median OC was $-0.11 \mathrm{~mm}$ (SD 0.20). These instances occurred within two datasets: all horizontal measurements, $5 / 7(71.4 \%)$ at $3.0 \mathrm{~mm}$ distance, and $4 / 7(57.1 \%)$ at $0.4 \mathrm{~mm}$ resolution.

Among paired measurements, UN was smaller than COR by $0.5 \mathrm{~mm}$ or more in two instances, with a maximum difference of $-0.64 \mathrm{~mm}$. Both instances were horizontal $0.5 \mathrm{~mm}$ measurements at $0.4 \mathrm{~mm}$ resolution in the same dataset, with mean OU of $0.50 \mathrm{~mm}$ (SD 0.20 ) and mean $\mathrm{OC}$ of $0.64 \mathrm{~mm}$ (SD 0.13).

No statistically significant effect on DIF was found for machine type $(\mathrm{p}=0.85)$, resolution ( $\mathrm{p}$ $=0.67)$, quadrant $(\mathrm{p}=0.74)$, cluster $(\mathrm{p}=0.68)$ or direction $(\mathrm{p}=0.34)$. 


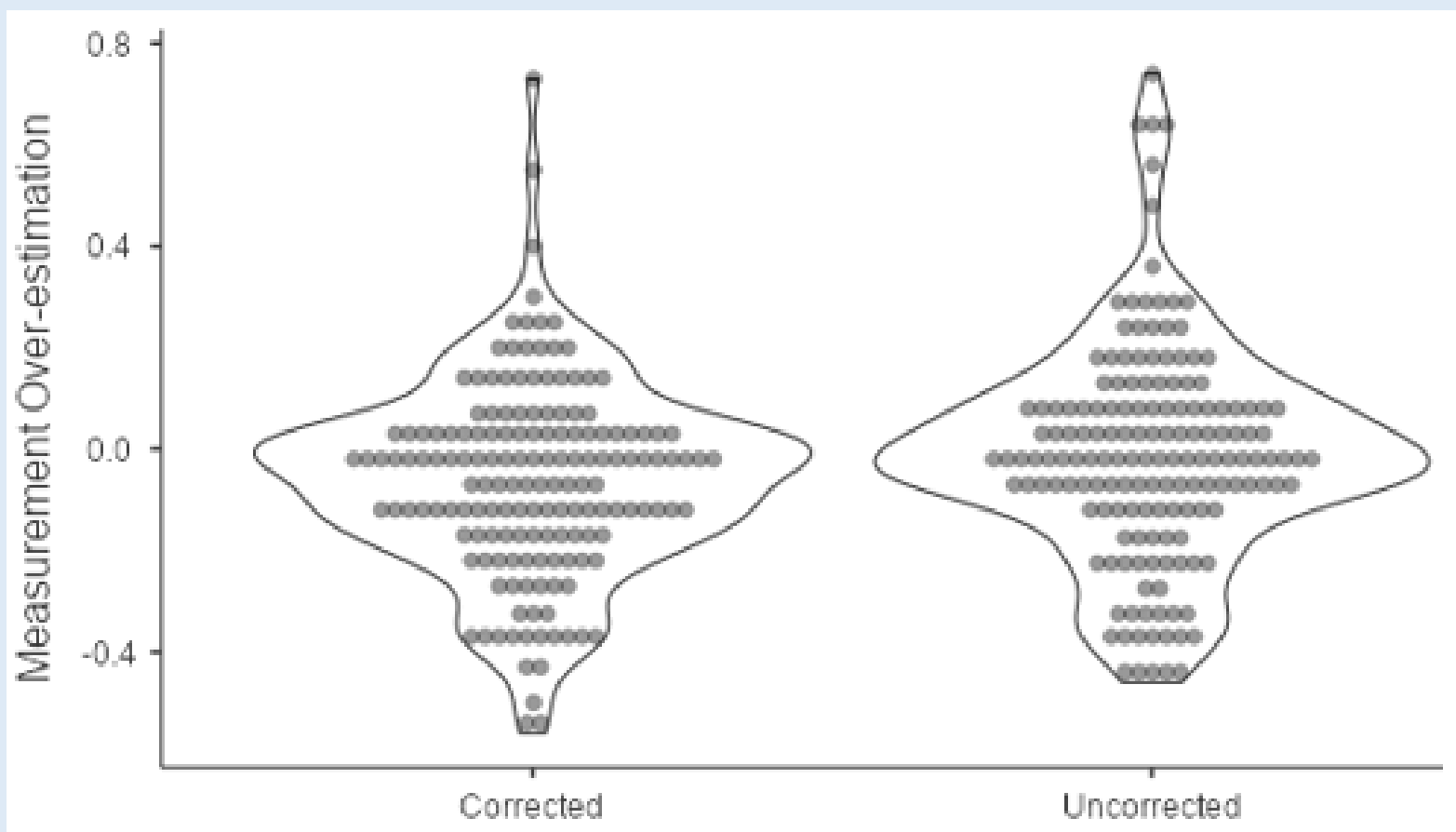

Dataset Orientation

Figure 3: Violin histogram of distance over-estimation split by viewing orientation.

\begin{tabular}{|c|c|c|c|c|c|}
\hline \multirow{2}{*}{$\begin{array}{c}\text { Distance } \\
(\mathbf{m m})\end{array}$} & \multicolumn{2}{|c|}{$\begin{array}{c}\text { Median Measured } \\
\text { Distance (and SD) }\end{array}$} & \multirow{2}{*}{$\begin{array}{c}\text { Difference in } \\
\text { Medians }^{\mathbf{A}}\end{array}$} & \multicolumn{2}{c|}{$\begin{array}{c}\text { Maximum Measured } \\
\text { Distance }\end{array}$} \\
\cline { 2 - 3 } \cline { 5 - 6 } & Uncorrected & Corrected & & Uncorrected & Corrected \\
\hline $0.5^{\mathrm{B}}$ & $0.52(0.19)$ & $0.54(0.19)$ & $0.01(0.06-0.08)$ & 0.77 & 0.68 \\
\hline 1 & $0.98(0.19)$ & $0.98(0.17)$ & $0.03(0.02-0.07)$ & 1.56 & 1.4 \\
\hline 2 & $1.95(0.22)$ & $1.95(0.14)$ & $-0.01(-0.07-0.06)$ & 2.63 & 2.15 \\
\hline 3 & $2.98(0.26)$ & $2.84(0.20)$ & $0.13(0.08-0.28)$ & 3.74 & 3.3 \\
\hline
\end{tabular}

Table 1: Distribution of measurements split by true distance between root and IDC, and effect of orientation correction. (A) Expressed as uncorrected minus corrected measurement, with $95 \%$ confidence interval; (B) $0.5 \mathrm{~mm}$ group comprises horizontal measurements only. 


\section{Discussion}

This study provides insight into whether correction of CBCT dataset alignment is beneficial, particularly in relation to linear measurements at short distances. Examination of the findings and limitations of the present study, and previous studies of CBCT measurement accuracy, help provide direction for future research in the field.

\section{Measurement Accuracy in Previous Studies, Calliper Measurements and Retest Reliability}

The accuracy of CBCT measurements of cadavers and dry osseous specimens have been investigated in multiple previous studies [13]. Brüllmann and Schulze note in their review that no study appears to have investigated accuracy in CBCT scans of live patients [10].

A 2018 systematic review by Fokas, et al., analysed CBCT linear measurements and error across studies considered relevant to pre-surgical implant planning [13]. The review included mostly cadaver and dry specimen studies, where digital callipers were the predominant gold standard [13]. Included studies variably reported absolute or relative error, either between groups or within pairs, with high levels of intra- and inter-observer reliability [13]. While reported measurement errors varied considerably and often exceeded $1 \mathrm{~mm}$, the mean measurement error was 'sub-millimetre' and not of statistical significance in the majority of studies [13].

While high levels of inter- and intra-observer reliability have been demonstrated for calliper measurements in previous studies, and high calliper precision is often reported, there are potential methodological issues with calliper measurements [13]. No attempt was made in this study to compare the dimensions of the model with digital calliper measurements, as it was anticipated that the larger component of measurement differences would result from errors in model sectioning or calliper angulation, rather than inherent model inaccuracy.

This study provides evidence for the practicality of highly accurate CAD-CAM models as a gold standard for future CBCT measurement accuracy studies. The accuracy to which the lathe was calibrated, as well as the immediate friction-fit between components on assembly and lack of air gap or other visible issues on a $0.076 \mathrm{~mm}$ CBCT scan were considered reliable evidence for the accuracy of the model dimensions in this study.

High levels of intra-observer reliability in this study are consistent with values reported in the relevant literature [13]. Inter-observer reliability was not assessed in this study due to practical limitations, and ideally would be included in future research. 


\section{Even Though Errors are Small and the Mean Benefit of Correction is Small, Correction may still Prove Beneficial}

Overall, the magnitude and distribution of measurement errors in the present study are considered consistent with ranges previously reported in the literature [13]. The tendency for all measurements to slightly under-estimate distances may relate to inherent dataset properties, viewing software, or systematic bias in operator technique.

The mean values obtained for of OU, OC and DIF were not considered sufficiently large to be 'clinically significant' in surgical planning. However, it is worth noting that individual overestimations were of greater magnitude than individual under-estimations, and occurred more frequently for uncorrected measurements.

The possibility remains that for CBCT scans of live patients, with additional factors such as movement, a $0.5 \mathrm{~mm}$ over-estimation due to non-correction may contribute to a 'clinically significant' distance over-estimation, either through simple additive or interaction processes. To discern these larger total over-estimations in live patient-based studies would require the inclusion of a gold standard reference of known dimensions within the scan.

\section{Influence of Machine Type}

While the effects of machine type on over-estimation were found to be statistically significant for both UN and COR, the mean differences were thought too small to be of clinical significance. The occurrence of over-estimations over $0.5 \mathrm{~mm}$ only within two Planmeca clusters, and under-estimations over $0.5 \mathrm{~mm}$ only within two iCAT clusters may relate to inherent machine differences. However, systematic difference in apparatus positioning differences in exposure parameters between machines may also account for the differences. The quantification of model positioning and angulation in future studies may help elucidate the relative contribution of machine/exposure versus positioning factors to these differences.

The absence of statistically significant differences in OU, OC or DIF by quadrant suggests that overall, the manufactured positioning jigs for each side were relatively similar, and operator measurement technique did not vary considerably when measuring on 'patient left' versus 'patient right' sides. 


\section{Influence of Scan Resolution}

While the effect of resolution on OU and OC was found to be statistically significant, in the context of surgical treatment planning, the small mean differences are not thought to be clinically significant. The sub-millimetre accuracy of measurements at a $0.4 \mathrm{~mm}$ voxel size supports the commentary by Fokas, et al., that measurements at $0.4 \mathrm{~mm}$ are sufficiently accurate for pre-surgical planning [13].

The tendency for measurements from datasets at $0.2 \mathrm{~mm}$ to be larger than at $0.4 \mathrm{~mm}$ may relate to greater 'blurring' of the interface between structures due to greater partial volume averaging, causing the structures to appear closer together.

\section{Influence of Measurement Direction}

There was a tendency for horizontal measurements to be larger than vertical measurements of the same distance. Horizontal measurements were made from an angulated root surface, while vertical measurements were made from the most inferior point of the root. Differences may be inherent in the way these interfaces were scanned and represented within the dataset, or in how they were ultimately perceived and measured. While the mean difference was small, this aspect needs further consideration.

\section{Possible Sources of Bias}

The possibility of unconscious bias in the current study is worthy of consideration. As the observer was not blinded to the first measurement prior to taking subsequent measurements, the possibility remains that subsequent measurements reflect knowledge of the error inherent in the first measurement. The use of known actual distances, and predictable nature of progression between slices, may have further facilitated a degree of unconscious measurement bias. It is not possible to retrospectively assess for differences between first and subsequent measurements in this study, given that the median value was the one recorded.

\section{Theoretical Maximum Resolution and Acquisition Alignment}

The design of the present study was such that the structures within the model were oriented obliquely to the detector elements during image acquisition, to reflect the position of equivalent structures during acquisition of real patient data. While this design better reflects a real-life patient scenario than orthogonal model alignment, and provides the basis for comparison of measurements in uncorrected and corrected views, more appropriate methods are available to assess maximal/theoretical spatial resolution [10]. 
No comparison was made in this study to models scanned with the IDC aligned along the orthogonal plane, or between different known angulations. While the literature in this area appears limited, a 2019 study by Koch, et al., assessed the dimensional accuracy of mandibles scanned at differing coronal and sagittal angulations (of up to $40^{\circ}$ ), utilising 'best fit' software [16]. In all instances, the differences in dimensional accuracy were deemed to be clinically insignificant [16].

\section{Effects of Scatter and Contrast Resolution on Measurement Accuracy}

Where measurement accuracy is reported in the literature, it is worth considering the different contributions of scatter to the resulting image between cadaver studies, dry osseous specimens and non-anatomic or machined structures. The finding of Lascala, et al., that CBCT measurements were less accurate in the skull base versus the mandible raises the possibility that increased scatter (e.g. from the dense skull base structures) may be an important factor in measurement accuracy [9]. To date, no direct comparisons in the accuracy of measurements between ex-vivo, cadaver or dry specimen scenarios have been published.

The age of cadaveric specimens is itself worthy of consideration. Increased numbers of dental restorations in older cadaveric specimens may result in increased scatter and artefacts and osteoporotic changes in the IDC cortical border may affect its use as a measurement landmark $[17,18]$. Further, while the general course and direction of the IDC does not appear to change with age, the relative position and mean distance between the IDC and tooth roots may change with age $[19,20]$. The use of live patients in research, where appropriate, would more readily allow for the inclusion of patients of varying age and restorative status; however, a number of practical and ethical factors must also be considered.

In the present study, the scatter created by the $120 \mathrm{~mm}$ plastic container is of lesser magnitude and more even distribution than that expected in a patient. Further, landmark selection was idealised by the high contrast resolution between the materials, clearly-defined structure margins, and lack of movement during acquisition. In studies utilising patients or anatomic specimens, landmark selection is expected to be more variable, given both the variability of normal patient anatomy, and potentially lower differences in attenuation at the junction between structures. Very minimal inadvertent patient movement during scan acquisition also becomes a factor with live patients, and is not reflected in cadaver studies.

Further, as CBCT reconstruction algorithms are designed for scanning of intact patients, including soft tissues, measurements obtained from scans of partial/dry specimens or model simulations may not accurately represent the spatial characteristics of in-vivo scenarios.

Golding R | Volume 2; Issue 2 (2021) | JDHOR-2(2)-029 | Research Article

Citation: Golding R, et al. Accuracy of CBCT Linear Measurements in Orthogonal versus Corrected

Coronal Planes. J Dental Health Oral Res. 2021;2(2):1-14.

DOI: http://dx.doi.org/10.46889/JDHOR.2021.2204 


\section{Directions for Future Research}

A future retrospective study comparing uncorrected and corrected measurements within CBCT scans of real patients could confirm differences between uncorrected and corrected measurements, however the lack of gold standard would prevent assessment of the true accuracy of measures for each view. A prospective study could be considered, allowing a model of known dimensions to be included in the scan. Such a study would allow assessment of real-world overall error for measurements in CBCT datasets, as well as the effects of orientation correction for patient scans.

\section{Conclusion}

This study describes a novel methodology for assessment of accuracy of CBCT measurements as it relates to viewing angulation. Despite potential small increases in absolute error, measurements from corrected views are associated with decreased risk of distance overestimation. As no research appears to have been undertaken on CBCT accuracy in live patients against a gold standard, further research in the area may include the use of models of known dimensions scanned in-vitro, to account for the effects of patient movement, scatter, and allow comparison with the use of true anatomic landmarks.

\section{Funding}

This work was supported by the Colgate-Palmolive Student Research Grant and by University Student Research Funding. Funding sources had no involvement in study design, data management, writing, or decision to publish.

\section{References}

1. Dudhia R, Monsour PA, Savage NW, Wilson RJ. Accuracy of angular measurements and assessment of distortion in the mandibular third molar region on panoramic radiographs. Oral Surg Oral Med Oral Pathol Oral Radiol Endod. 2011;111:508-16.

2. Bell GW. Use of dental panoramic tomographs to predict the relation between mandibular third molar teeth and the inferior alveolar nerve: Radiological and surgical findings, and clinical outcome. Br J Oral Maxillofac Surg. 2004;42:21-7.

3. Blaeser BF, August MA, Donoff RB, Kaban LB, Dodson TB. Panoramic radiographic risk factors for inferior alveolar nerve injury after third molar extraction. J Oral Maxillofac Surg. 2003;61:417-21.

4. Bell GW, Rodgers JM, Grime RJ, Edwards KL, Hahn MR, Dorman ML, et al. The accuracy of dental panoramic tomographs in determining the root morphology of mandibular third molar teeth before surgery. Oral Surg Oral Med Oral Pathol Oral Radiol Endod. 2003;95:119-25.

5. Friedland B, Donoff B, Dodson TB. The use of 3-dimensional reconstructions to evaluate the anatomic relationship of the mandibular canal and impacted mandibular third molars. J Oral Maxillofac Surg. 2008;66:1678-85.

Golding R | Volume 2; Issue 2 (2021) | JDHOR-2(2)-029 | Research Article 
6. Radiation Protection No. 172 - CBCT for dental and maxillofacial surgery: Evidence-based guidelines (The SEDENTEXCT Project). Brussels: European Commission. 2012. [Last accessed on July 15, 2021] http://www.sedentexct.eu/files/radiation_protection_172.pdf

7. Mozzo P, Procacci C, Tacconi A, Tinazzi Martini P, Bergamo Andreis IA. A new volumetric CT machine for dental imaging based on the cone-beam technique: preliminary results. Eur Radiol. 1998;8:1558-64.

8. Nemtoi A, Czink C, Haba D, Gahleitner A. Cone beam CT: a current overview of devices. Dentomaxillofac Radiol. 2013;42:20120443.

9. Lascala CA, Panella J, Marques MM. Analysis of the accuracy of linear measurements obtained by cone beam computed tomography (CBCT-NewTom). Dentomaxillofac Radiol. 2004;33:291-4.

10. Brüllmann D, Schulze RKW. Spatial resolution in CBCT machines for dental/maxillofacial applications-what do we know today? Dentomaxillofac Radiol. 2015;44:20140204.

11. Butt A, Mahoney M, Savage N. The impact of computer display performance on the quality of digital radiographs: a review. Aust Dent J. 2012;57:16-23.

12. Baumgaertel S, Palomo JM, Palomo L, Hans MG. Reliability and accuracy of cone-beam computed tomography dental measurements. Am J Orthod Dentofac Orthop. 2009;136:19.

13. Fokas G, Vaughn VM, Scarfe WC, Bornstein MM. Accuracy of linear measurements on CBCT images related to presurgical implant treatment planning: A systematic review. Clin Oral Implants Res. 2018;29:393415.

14. Momin M, Matsumoto K, Ejima K, Asaumi R, Kawai T, Arai Y, et al. Correlation of mandibular impacted tooth and bone morphology determined by cone beam computed topography on a premise of third molar operation. Surg Radiol Anat. 2013;35:311-8.

15. Mahasantipiya PM, Savage NW, Monsour PAJ, Wilson RJ. Narrowing of the inferior dental canal in relation to the lower third molars. Dentomaxillofac Radiol. 2005;34:154.

16. Koch GK, Hamilton A, Wang K, Herschdorfer L, Lee KH, Gallucci GO, et al. Dimensional accuracy of cone beam CT with varying angulation of the jaw to the X-ray beam. Dentomaxillofac Radiol. 2019;48:20180319.

17. Timock AM, Cook V, McDonald T, Leo MC, Crowe J, Benninger BL, et al. Accuracy and reliability of buccal bone height and thickness measurements from cone-beam computed tomography imaging. Am J Orthod Dentofac Orthop. 2011;140:734-44.

18. Suomalainen A, Ventä I, Mattila M, Turtola L, Vehmas T, Peltola JS. Reliability of CBCT and other radiographic methods in preoperative evaluation of lower third molars. Oral Surg Oral Med Oral Pathol Oral Radiol Endod. 2010;109:276-84.

19. Simonton JD, Azevedo B, Schindler WG, Hargreaves KM. Age- and gender-related differences in the position of the inferior alveolar nerve by using cone beam computed tomography. J Endod. 2009;35:944-9.

20. Littner MM, Kaffe I, Tamse A, Dicapua P. Relationship between the apices of the lower molars and mandibular canal-a radiographic study. Oral Surg Oral Med Oral Pathol Oral Radiol Endod. 1986;62:595602. 